\title{
A Comparison of the Effects of Intravenous Pentagastrin on Patients with Social Phobia, Panic Disorder and Healthy Controls
}

Una D. McCann, M.D., Shiyoko O. Slate, B.A., Marilla Geraci, M.S.N., Diana Roscow-Terrill, M.S., and Thomas W. Uhde, M.D.

The present study sought to determine whether social phobics, like patients with panic disorder, have increased sensitivity to the panicogenic effects of pentagastrin. Intravenous pentagastrin and placebo were administered in a double-blind fashion to 19 social phobics, 11 patients with panic disorder, and 19 healthy controls while they participated in a structured social interaction task. Behavioral, cardiovascular, and neuroendocrine responses were obtained. Pentagastrin led to panic attacks in $47 \%$ of

KEY WORDS: CCK; Chemical models; Panic; Social interaction

A growing body of preclinical and clinical evidence suggests that central nervous system cholecystokinin (CCK) is involved in the modulation of anxiety. CCK receptors are present in high concentrations in the limbic system and other brain regions thought to mediate anxiety (Crawley 1985), and central administration of CCK agonists leads to anxious behaviors in a variety of animal species (Fekete et al. 1984; Ervin et al. 1991;

From the Lnit on Anxiety Disorders (UDM, SOS, MG, DR-T), Biological Psychiatry Branch, NIMH, NIH, Bethesda, MD; and the Department of Psychiatry (TWU), Wayne State University Medical School, Detroit, MI.

Address correspondence to: Dr. Una McCann, Unit on Anxiety Disorders, Biological Psychiatry Branch, NIMH, NIH, Building 10: Room 3N/212, MSC 1272, 10 Center Drive, Bethesda, MD 20892-1272.

Received April 2, 1996; revised June 19, 1996; accepted August 14, 1996 the social phobics, $64 \%$ of the panic disorder patients, and $11 \%$ of the healthy controls. The social interaction itself increased anxiety, blood pressure, and pulse in all three groups. These findings suggest that the panicogenic effects of pentagastrin are not limited to patients with panic disorder and provide further evidence for shared neurobiology in social phobia and panic disorder. (c) 1997 American College of Neuropsychopharmacology [Neuropsychopharmacology 16:229-237, 1997]

Harro and Vasar 1991; Harro et al. 1993). In humans, peripheral administration of CCK-4, a naturally occurring fragment and CCK receptor agonist, induces doserelated increases in anxiety and symptoms of panic (Bradwejn et al. 1990, 1991a, 1991b). Furthermore, patients with panic disorder are more sensitive to the panic-inducing effects of CCK-4 and pentagastrin, a synthetic pentapeptide analog of CCK-4 than nonanxious control subjects (Bradwejn et al. 1991a; Abelson and Nesse 1994; van Megen et al. 1994).

Social phobia, a prevalent anxiety disorder, has been estimated to be the second most common mental illness in the United States (Kessler et al. 1994). Patients with generalized social phobia, the most severe form of social phobia, are unable to perform routine social and occupational activities because of intense anxiety and consequent avoidance. Although growing research interest has led to increased understanding of the genetics (Fyer et al. 1990, 1993; Kendler et al. 1992, 1995), epidemiology (Regier et al. 1988; Kessler et al. 1994), and phenomenology (Uhde et al. 1991) of social phobia, it is never- 
theless the least studied of all the anxiety disorders. For example, little is known about the neurobiological substrates of social phobia and to what extent they overlap with those of panic disorder. Because many CCK-4and pentagastrin-induced symptoms, such as severe anxiety, flushing, and abdominal discomfort, also are symptoms of social phobia, it would be of interest to determine whether the anxiogenic effects of CCK agonists found in patients with panic disorder generalize to patients with social phobia. If so, this would provide further evidence for a role of CCK in anxiety and would shed light on the comparative neurobiology of social phobia and panic disorder.

There have been three published studies that used pharmacological challenges to compare patients with social phobia and panic disorder. In the first study (Liebowitz et al. 1985), lactate was administered to patients with social phobia and patients with panic disorder and their behavioral responses were compared. Unlike patients with panic disorder, patients with social phobia did not report lactate-induced panic, suggesting that the two disorders are distinct. A second study used oral caffeine as the pharmacological challenge, comparing the behavioral responses of patients with social phobia and with panic disorder against those of healthy controls (Tancer et al. 1991). Both patient groups had higher rates of caffeine-induced panic than the control group, although the rate of caffeine-induced panic in subjects with panic disorder was significantly lower $(27 \%)$ than previously found (Uhde 1990). In a third study (Tancer et al. 1994), patients with social phobia and patients with panic disorder were both found to have blunted clonidine-induced growth hormone responses when compared to healthy volunteer subjects, although growth hormone responses in social phobics tended to be higher (less blunted) than those in patients with panic disorder. This finding was interpreted as an indication of possible noradrenergic dysregulation in both panic disorder and social phobia. Although results from these studies are somewhat conflicting, they suggest that there may be some overlap in the neurobiology of social phobia and panic, although the nature and extent of the overlap are unclear.

The purpose of the present study was to compare the behavioral and physiological effects of intravenous pentagastrin in patients with social phobia, panic disorder, and healthy volunteers in an effort to further evaluate the role of CCK in anxiety and provide information on features shared by social phobia and panic disorder. It was hypothesized that pentagastrin, administered in a contextually relevant setting, would lead to increases in syndrome-specific symptoms in patients with social phobia. It was further hypothesized that successful provocation of syndrome-specific symptoms in social phobia would facilitate comparison of the behavioral and physiological correlates of social phobia and panic disorder.

\section{METHOD}

\section{Subjects}

Nineteen patients with generalized social phobia, 11 patients with disorder, and 19 healthy controls were recruited to participate in this study. Subjects were between the ages of 18 and 55 . All subjects were in good general health as determined by medical history, physical exam, electrocardiogram, and blood and urine chemistries, including a complete blood count, liver and thyroid function tests, hepatitis and HIV screens, routine urinalyses and urine drug screens for therapeutic and illicit drugs. Presence (or absence) of DSM-III-R Axis I psychiatric diagnosis was determined using a structured psychiatric interview (SADS-LA(R); Schleyer et al. 1990]. Some subjects in both patient groups had comorbid diagnoses in addition to their chief complaint. Patients with both panic disorder and social phobia were assigned to the panic disorder group. Because patients with panic disorder already have been shown to have increased susceptibility to the panicogenic effects of pentagastrin, assignment of patients with comorbid social phobia to the panic group minimized the likelihood of falsely inflating the anxiogenic effects of pentagastrin in the social phobia group. Pregnant women were excluded (all women were studied during the first 10 days of their menstrual cycle). Subjects were recruited through advertisements in local and college newspapers, advertisements placed on bulletin boards, and through an institutional Normal Volunteer Office. Subjects provided written informed consent prior to study participation and agreed to refrain from ingesting caffeine, alcohol, and any medications for 2 weeks prior to study. In addition, subjects were instructed to maintain a low-monoamine diet for 3 days prior to study and fasted after midnight on the morning of each study day.

\section{Design}

Subjects came to an outpatient psychiatry clinic for two separate challenge sessions separated by at least 2 days. At approximately 9:30 A.M. (-120 minutes, 120 minutes prior to the infusion procedure) on each study day, an intravenous catheter was placed in a forearm vein for repeated blood draws and for pentagastrin (or placebo) infusion. On the other arm, an automated blood pressure cuff (Dinamapp, Critikon, Tampa, FL) was placed to monitor blood pressure and pulse. Subjects remained seated in a partially reclined chair (70 degrees) for the entire procedure. Immediately after IV insertion, a curtain was drawn between the patient and the IV apparatus, the vital signs display monitor, and the research nurse so that subjects were unaware of when blood draws were taking place, when drug was infused, and the effect of the various experimental manipulations on 
vital signs. Shortly after IV insertion, a research associate reviewed instructions for a structured social interaction task (role play), described in detail below. One hour after IV insertion ( -60 minutes), patients completed a baseline rating packet that included a Clinician-Rated Zung Anxiety Scale (Zung 1971), Visual Analog Scales (VAS) for anxiety and panicky feelings, the Speilberger State Anxiety Scale (Speilberger et al. 1970), a DSM-IV panic symptom checklist, and a side effect checklist. Seventy-five ( -45 minutes), 90 (-30 minutes), and 105 ( -15 minutes) minutes after IV insertion, baseline blood samples were collected and patients completed the VAS for anxiety and self-consciousness. At time 0 ( 0 minutes) the patient began a five-minute structured social interaction task.

Two blood samples were collected prior to administration of pentagastrin, 1 minute and 45 seconds into the social interaction task $(+1$ minute 45 seconds). Pentagastrin, at a dose of $0.6 \mu \mathrm{g} / \mathrm{kg}$ (or normal saline at an equal volume of $20 \mathrm{cc}$ ), was administered in a double-blind fashion over $60 \mathrm{~s}$. Pentagastrin and placebo were administered in random order. The dose of pentagastrin was selected based on findings from two previous clinical studies demonstrating that the $0.6-\mu \mathrm{g} / \mathrm{kg}$ dose is effective in inducing panic attacks in patients with panic disorder (Abelson and Nesse 1994; van Megen et al. 1994) and because a previous dose-response study in healthy controls indicated that this dose produced nearmaximal behavioral effects without producing untoward symptoms (McCann et al. 1995). Following completion of pentagastrin infusion, the IV line was flushed with $10 \mathrm{cc}$ normal saline ( +2 minutes 45 seconds to +3 minutes), and three additional blood samples were obtained in succession (approximately +3 minutes to +6 minutes).

Immediately after the completion of the role play ( +5 minutes), the patients were asked to complete the VAS for anxiety and self-consciousness, retrospectively rating sensations that they experienced during the most intense portion of the social interaction task (the "peak" time point) and another set of VAS referring to their sensations at that moment (the "post" time point). They then completed a larger packet of retrospective questionnaires (the same packet as that completed at baseline) corresponding to their "peak" experiences during the role play. It typically took a subject 5 minutes to complete all of the "peak" and "post" social interaction task ratings. Subsequent blood samples and VAS for anxiety and self-consciousness were collected thirty minutes $(+30)$, sixty minutes $(+60)$, ninety minutes $(+90)$, and two hours $(+120)$ after the initiation of the social interaction task.

\section{Structured Social Interaction Task}

As indicated, subjects received pentagastrin while participating in a structured social interaction task, as pre- viously described (McCann et al. 1995). This paradigm was used for three reasons. First, by asking all subjects to engage in an identical task, an added element of experimental control was introduced, presumably decreasing variability that is inherent in nonstructured infusion procedures. Second, because one subject group consisted of patients with generalized social phobia, it seemed appropriate to provide a contextually relevant setting for the challenge to optimize the possibility of producing syndrome-specific symptoms (such a relevant setting is not necessary in pharmacological challenges in patients with panic disorder, since by definition, panic attacks sometimes occur spontaneously) in that disorder. Finally, because the social interaction task produces anxiety even in the absence of a pharmacological challenge (McCann et al. 1995), this particular behavioral paradigm is a useful method for "filtering out" anxiety stemming from nonspecific sources, so that differences in anxiety between the pentagastrin and placebo challenge days can be reasonably attributed to the pharmacological effects of pentagastrin.

The structured interaction task involved a role-play where subjects were told to imagine that they were at a dinner party and that they would be seated next to a stranger of the opposite sex (the confederate), who entered the room immediately prior to the social interaction task. The stranger was described as reserved, and thus it would be the subject's responsibility to initiate and maintain a 5-minute conversation. The entire roleplay was videotaped, with the video camera in full view of the subject. Separate confederates were used for the pentagastrin and placebo challenge days. The confederate was blind to the patient's diagnosis.

\section{Physiological Measures}

Blood samples were drawn 30,15, and 1 minutes prior to pentagastrin (or placebo) infusion. Three consecutive blood samples were taken after pentagastrin infusion, with subsequent blood draws at 30,60, and 90 minutes postinfusion. Blood samples were immediately placed on ice and were subsequently centrifuged for $10 \mathrm{~min}$ utes at 3,600 rpm for plasma separation. Plasma samples were stored in a $-70^{\circ} \mathrm{C}$ freezer for subsequent plasma cortisol assay. Measures of blood pressure and pulse were taken at the time of each blood draw and every 2 minutes during the role-play.

\section{Statistical Analyses}

Data were analyzed by repeated-measures ANOVA with Greenhouse-Geisser corrections. Results were considered significant at a level of $p<.05$. When significant main effects of drug or time, or significant drug $\times$ time interactions were observed, Bonferroni post hoc tests were performed at individual time points to determine 
which groups differed significantly. All statistical analyses were carried out using SPSS for Windows (SPSS, Chicago, IL).

For their symptoms to be defined as a panic attack, patients needed to report that they experienced a panic attack plus at least four of the 13 symptoms listed in DSM-IV criteria for panic, in moderate to severe intensity. Because gastrointestinal symptoms following pentagastrin are expected, when stomach discomfort was reported, four additional symptoms were required for classification as a panic attack. Furthermore, only one of the symptoms reported could be "fear of dying" or "fear of going crazy" (i.e., if a patient reported both of these symptoms, it was counted only once, and three other symptoms also were required). The method for assessing panic attack symptoms was changed early in the course of the protocol from a 100-mm VAS to an ordinal questionnaire where symptoms were rated from absent (1) to severe (4). This was done for two reasons. First, it allowed us to compare findings from the present study to those in a parallel study in which patients receive pentagastrin during sleep, where use of a VAS is impractical because of darkness. Second, it enabled us to rate patients on panic attack symptoms orally in situations where patients were unable to complete the questionnaire manually. For data analysis of the present study, visual analog ratings were converted to an ordinal scale in the following manner: Ratings between 0 and $40 \mathrm{~mm}$ were designated as mild (2), those between 40 and 60 as moderate (3), and those between 60 and 100 as severe (4).

Analysis of pentagastrin-induced side effects was conducted by totaling all side effect ratings and averaging the ratings within each diagnostic group. These averages were then compared using repeated-measures ANOVA with Bonferroni post hoc analyses.

\section{RESULTS}

\section{Epidemiological Data}

Subjects in the three experimental groups were similar in age, tending to be in their early to mid-thirties. The male/female ratio in the social phobia group and healthy volunteer group was similar $(17 / 2$ and $16 / 3$, respectively), but the panic disorder group was more evenly distributed across gender lines (male/female = $6 / 5$ ). Subjects with social phobia and controls were more likely to be married than those panic disorder and as a group, had slightly higher levels of education.

The majority of subjects in both patient groups had at least one comorbid lifetime diagnosis. However, patients with panic disorder were more likely than those with social phobia to have more than one comorbid lifetime diagnosis (average number of comorbid diagnosis, $3.54 \pm 1.13$ versus $1.53 \pm 3.04$, respectively). As expected, social phobics were younger when they experi- enced the onset of their illness than patients with panic disorder $(12.32 \pm 5.01$ versus $22.9 \pm 8.81$ years of age, respectively).

\section{Behavioral Effects}

Panic Attacks. Nine of 19 (47\%) patients with social phobia, 7 of $11(64 \%)$ patients with panic disorder and 2 of $19(11 \%)$ healthy volunteers developed panic attacks following pentagastrin infusion. No subject developed panic following placebo administration. All panic attacks occurred and resolved within the $31 / 4$ minutes from the initiation of pentagastrin infusion until the end of the social interaction task. Chi-Square analysis indicated a significant group difference ( $\mathrm{Chi}=9.97, p=.007$ ). A Chi-Square multiple comparisons procedure indicated that both patient groups were more likely to have pentagastrin-induced panic than healthy controls $(p<$ .05 ), but that the two patient groups were not different with regard to the likelihood for panic. The symptoms of pentagastrin-induced symptoms and pentagastrininduced panic attacks in the three groups are illustrated in Table 1.

Table 1. Panic Symptoms after Pentagastrin Infusion ${ }^{i}$

\begin{tabular}{|c|c|c|c|}
\hline & $\begin{array}{c}\text { Panic } \\
\text { Disorder }\end{array}$ & $\begin{array}{l}\text { Social } \\
\text { Phobia }\end{array}$ & $\begin{array}{l}\text { Normal } \\
\text { Controls }\end{array}$ \\
\hline All subjects & $n=11$ & $n=18$ & $n=18$ \\
\hline Trouble Breathing & $2.18 \pm 0.87$ & $2.44 \pm 0.78$ & $2.17 \pm 0.79$ \\
\hline Palpitations & $.64 \pm 1.03$ & $2.72 \pm 0.75$ & $1.94 \pm 0.73$ \\
\hline Chest pain & $.36 \pm 1.03$ & $2.33 \pm 0.84$ & $2.06 \pm 0.73$ \\
\hline Choking & $2.45 \pm 1.04$ & $2.39 \pm 0.85$ & $1.89 \pm 0.58$ \\
\hline Dizziness & $2.73 \pm 0.79$ & $2.61 \pm 0.92$ & $1.89 \pm 0.47$ \\
\hline Numbness & $2.73 \pm 1.10$ & $2.50 \pm 0.79$ & $2.00 \pm 0.59$ \\
\hline Hot/cold flashes & $2.64 \pm 0.92$ & $2.72 \pm 1.02$ & $2.17 \pm 0.62$ \\
\hline Sweating & $2.73 \pm 1.01$ & $2.56 \pm 0.62$ & $2.11 \pm 0.76$ \\
\hline Trembling & $2.73 \pm 1.01$ & $2.50 \pm 0.51$ & $2.00 \pm 0.69$ \\
\hline Feelings of unreality & $2.64 \pm 1$ & $2.50=1$ & $1.94 \pm 0.73$ \\
\hline Fear of dying & $2.27 \pm 0.79$ & $2.06 \pm 0$ & \\
\hline Fear of go & $2.55 \pm 0.93$ & $2.22 \pm 0.81$ & $2.00 \pm 0.77$ \\
\hline Mean & $2.55 \pm 0.66$ & $2.46 \pm 0.50$ & $2.00=$ \\
\hline Panic attack subjects & $n=7$ & $n=9$ & \\
\hline Trouble breathing & $2.67 \pm 0.82$ & $3.00 \pm 0.76$ & $4.00 \pm 0.00$ \\
\hline Palpitations & $3.17 \pm 1.17$ & & \\
\hline Chest pain & $2.83 \pm 1.17$ & $2.75 \pm 0.89$ & $3.50 \pm 0.71$ \\
\hline Choking & $3.17 \pm 1.17$ & $2.88 \pm 0.83$ & $2.00 \pm 1.41$ \\
\hline Dizziness & $3.00 \pm 0.89$ & $3.13 \pm 0.99$ & $1.50 \pm 0.71$ \\
\hline Numbness & $3.00 \pm 0.89$ & $3.00 \pm 0.93$ & $1.50 \pm 0.71$ \\
\hline Hot/cold flashes & $3.17 \pm 0.75$ & $3.00 \pm 1.07$ & $1.50 \pm 0.71$ \\
\hline Sweating & $2.83 \pm 1.17$ & $2.88 \pm 0.64$ & $3.00 \pm 1.41$ \\
\hline Trembling & $3.17 \pm 1.17$ & $2.63 \pm 0.52$ & $3.00 \pm 0.00$ \\
\hline Feelings of unreality & $3.17 \pm 1.17$ & $3.13 \pm 1.25$ & $3.50 \pm 0.71$ \\
\hline Fear of dying & $2.50 \pm 1.05$ & $2.25 \pm 0.89$ & $2.00 \pm 1.41$ \\
\hline Fear of going crazy & $3.00 \pm 1.10$ & $2.50 \pm 0.89$ & $3.50 \pm 0.71$ \\
\hline Mean severity & $2.97 \pm 0.18$ & $2.84 \pm 0.31$ & $2.54 \pm 0.18$ \\
\hline
\end{tabular}

"Ratings from VAS and ordinal DSM-IV Panic Attack Symptom Scales. 
Zung Anxiety Scale. (Figure 1) Main effects of diagnosis $[F(2,41)=18.34, p<.001]$, drug $[F(1,41)=47.59, p<$ $.001]$, and time $[F(1,41)=108.71, p<.001]$ were observed. In addition, interactions between drug and time $[F(1,41)=84.22, p<.001]$ and diagnosis and drug and time $[F(2,41)=6.68, p=.003]$ were evident. These differences reflected significantly higher anxiety on pentagastrin than on placebo days, with peak anxiety occurring during the role-play on the pentagastrin day. Post hoc testing indicated that both patient groups were more anxious than healthy controls on both challenge days, but that there were no differences between patients with panic disorder and those with social phobia on either challenge day.

Speilberger State Anxiety Scale. (Not shown) Significant main effects of diagnosis $[F(2,43)=35.69, p<.001]$, drug $[F(1,43)=9.44, p=.004]$, and time $[F(1,43)=97.47$, $p<.001]$ were evident. There also was an interaction between drug and time $[F(2,43)=13.75, p=.001]$. Both the social interaction task itself (i.e., placebo infusion) and pentagastrin infusion led to increased anxiety, although anxiety following pentagastrin infusion was significantly higher than that seen following placebo infusion. The two patient groups reported higher levels of anxiety than healthy volunteers on both challenge days, but there were no differences found between the two patient groups.

VAS, Anxiety. (See Figure 1.) Main effects of diagnosis $[F(2,40)=18.04, p<.001]$, drug $[F(1,40)=11.98, p=$ $.001]$, and time $[F(4,160)=161.47, p<.001]$ were seen on this analog scale, as were interactions of diagnosis by time $[F(8,160)=7.00, p<.001]$, drug by time $[F(4,160)=$ $14.91, p<.001]$, and diagnosis by drug by time $[F(8,160)=$ $3.04, p=.015]$. As was found with the Spielberger Anxiety Scale, anxiety increased significantly following placebo and pentagastrin infusions, although scores following pentagastrin were significantly greater than those following placebo. Post hoc testing revealed that the two patient groups reported significantly greater anxiety than control subjects at the peak time point (i.e., the time point retrospectively rated as "peak" intensity during the 5-minute social interaction task/infusion procedure) and immediately after completion of the social interaction (the "post" time point) on both placebo
PLACEBO
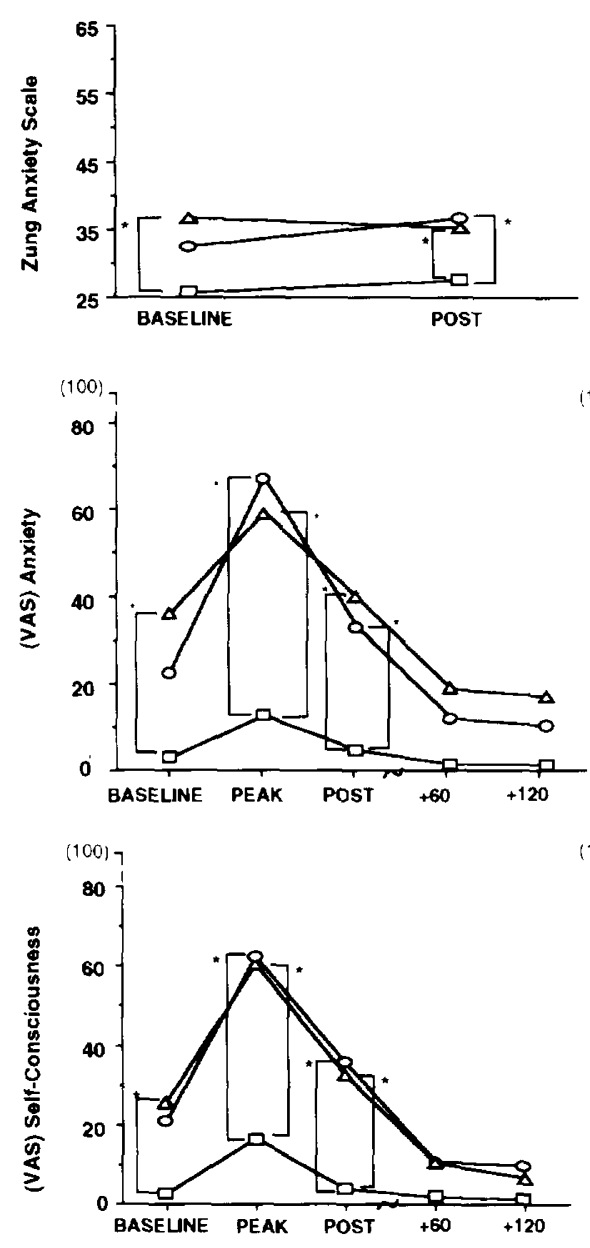

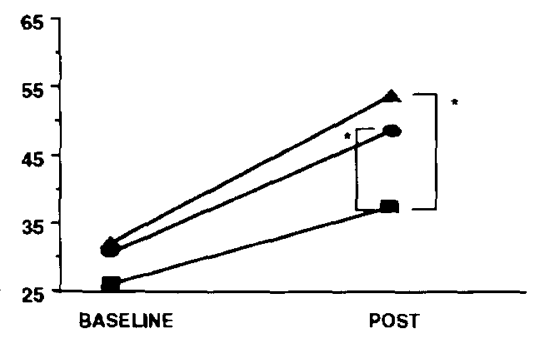

PENTAGASTRIN
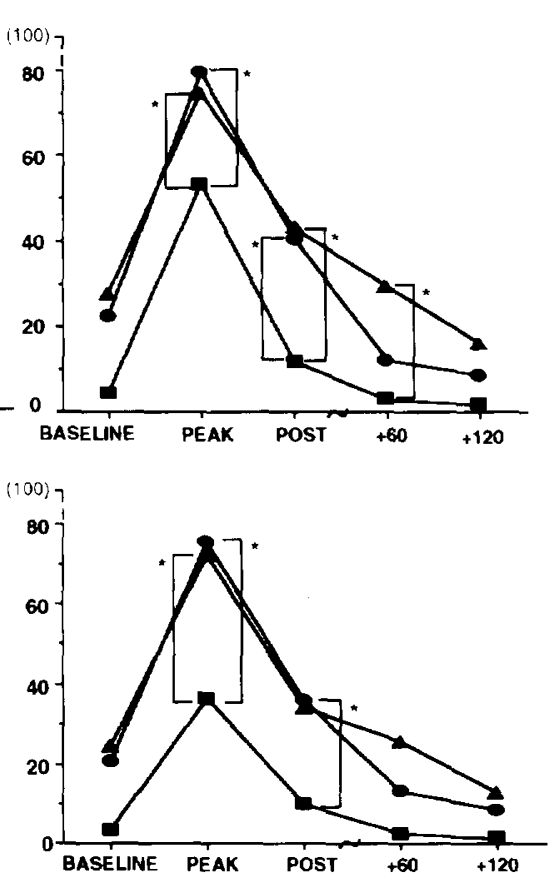

Figure 1. Self-rated measures of anxiety in patients with social phobia, patients with panic disorder, and healthy volunteers following infusions of placebo and pentagastrin. Peak, group means of the most intense symptoms experienced during the social interaction task/infusion procedure. Post, group means of symptom severity immediately after concluding the social interaction task. Triangles, panic disorder $(n=11)$; circles, social phobia $(n=18)$; squares, controls $(n=19)$. ${ }^{*}$ Significant differences at $p<0.05$ as measured by Bonferroni post hoc tests. 
and pentagastrin challenge days, with increases in anxiety following placebo undoubtedly reflecting the effects of the social interaction. Subjects with panic disorder also were significantly more anxious than healthy volunteers 60 minutes after pentagastrin infusion. There were no differences between patient groups at any time point.

VAS, Self-Consciousness. (See Figure 1.) Significant main effects of diagnosis $[F(2,40)=14.44, p<.001]$, drug $[F(1,40)=6.21, p=.017]$, and time $[F(4,160)=139.01, p=$ $.001]$ were observed. Interactions were also found between diagnosis by time $[F(8,160)=9.16, p=.001]$ and drug by time $[F(4,160)=4.52 . p=.013]$. As might be expected, the social interaction task alone (i.e., placebo infusion) led to increased self-consciousness. Pentagastrin enhanced this effect significantly. Post hoc testing revealed that the two patient groups were more self-conscious than control subjects at the peak time point during the social interaction task/infusion procedure and immediately after completion of the social interaction task (the "post" time point) on both placebo and pentagastrin challenge days. As with other measures of anxiety previously discussed, there were no differences between the two patient groups at any time point.

VAS, "Panicky" Feelings. (Not shown) Main effects of diagnosis $[F(2,45)=17.68, p<.001]$, drug $[F(1,45)=$ $11.9, p=.001]$, and time $[F(1,45)=79.05, p<.001]$ were seen. Interactions were found between diagnosis by time $[F(2,45)=5.25, p=.009]$ and drug by time $[F(1,45)=$ $28.72, p<.001]$. Post hoc tests revealed significant differences between the two patient groups and controls at the peak time point (i.e., during 5-minute social interaction task/drug infusion procedure) after both placebo and pentagastrin infusions. Within-group analysis revealed that social phobics had significantly more panicky feelings (though no panic attacks) after both placebo and pentagastrin infusion, whereas controls and patients with panic disorder had significant increases only after pentagastrin challenge.

Side Effect Questionnaire. When the three groups were compared on average side effect scores, main effects of diagnosis $[F(2,43)=17.38, p<.001]$, drug $[F(1,43)=$ $14.28, p<.001]$, and time $[F(1,43)=19.99, p<.001]$ were found. An interaction of drug by time $[F(1,43)=36.08, p<$ $.001]$ was observed. These differences reflected an increase in side effects following both placebo and pentagastrin, with a greater number of symptoms following pentagastrin. Post hoc tests revealed that patients with panic disorder reported a greater number of side effects than controls at both baseline and following infusion with either placebo or pentagastrin. Post hoc tests also revealed a difference between patients with panic disorder and social phobia, with patients with panic disorder reporting more symptoms following pentagastrin.
Both patient groups had significantly more symptoms than healthy controls.

\section{Physiological Effects}

Cardiovascular Responses. (See Figure 2.) Significant main effects of drug and time were seen on measures of pulse $[F(1,43)=10.81, p=.002 ; F(3,129)=92.13, p<$ .001 , respectively]. A drug by time interaction $[F(3,129)=$ $20.77, p<.001]$ also was observed. Pulse rates increased significantly during the social interaction task (i.e., following placebo) and increased further following pentagastrin. Measures of mean arterial pressure showed a main effect of time $[F(3,129)=129.25, p<.001]$ and a trend toward a main effect of diagnosis $[F(2,43)=2.88$, $p=.067$, indicating an increase in blood pressure associated with the role-play and a tendency for patients with panic disorder to have higher mean arterial blood pressures than those with social phobia and healthy controls.

Cortisol Measures. (See Figure 2.) Significant main effects of drug and time were found for cortisol concentrations $[F(1,36)=20.78, p<.001 ; F(4,144)=43.42, p<.001$, respectively], reflecting pentagastrin-induced rises in cortisol. Peak cortisol levels were seen 30 minutes after pentagastrin infusion. There were no differences in cortisol levels among groups.

\section{DISCUSSION}

The major finding of this study is that patients with generalized social phobia, like patients with panic disorder (Abelson and Nesse 1994; van Megen et al. 1994), are more likely than healthy controls to experience panic following pentagastrin, when pentagastrin is given in the context of a structured role-play. Following pentagastrin infusion, the two groups could not be distinguished by any measure of anxiety evaluated, including self-consciousness and panicky feelings. Whereas patients with panic disorder had slightly higher rates of pentagastrin-induced panic attacks than patients with social phobia ( $64 \%$ versus $47 \%$ ), this difference was not statistically significant. The strong anxiogenic effect of pentagastrin observed in patients with social phobia lend support to the view that the anxiogenic effects of CCK-4 and pentagastrin previously reported in patients with panic disorder (Bradwejn et al. 1990, 1991b; Abelson and Nesse 1994; van Megen et al. 1994) are likely due to pharmacological activity at CCK receptors rather than to nonspecific drug effects. In addition, they provide further evidence for overlap in the neurobiological substrates underlying the symptoms of panic disorder and social phobia. 
Although there were striking similarities between patients with panic disorder and those with social phobia in their responses to pentagastrin, there also were some differences. For example, patients with panic disorder reported a greater severity and number of pentagastrin-induced side effects than patients with social phobia. Furthermore, patients with panic disorder had a greater number of physical and neuropsychiatric symptoms than control subjects at baseline, whereas social phobics were similar to controls on this measure. These findings are in keeping with the notion that patients with panic disorder are keenly aware of alterations in bodily sensations but do not support the view that presence of physical symptoms is the source of panic, as social phobics also developed pentagastrin-induced panic attacks.

The infusion paradigm used in the present study differs from those used in previously reported studies of CCK agonists and could possibly account for the striking similarities seen in the two patient groups. Specifically, in the present study, pentagastrin was given in the context of a social interaction task, a task that is anxiety provoking even in healthy volunteers and one that is syndromally appropriate for patients with social phobia. Use of this paradigm might have provided an environment that enhanced the likelihood of patients with social phobia to experience syndromally specific symptoms, even though no patient experienced panic secondary to the social interaction alone (i.e., following placebo). Indeed, the social interaction task itself (i.e., in conjunction with placebo infusion) was quite anxiogenic and discriminated the two patient groups from controls quite well. The large anxiogenic effect of the social interaction task in both patient groups undoubtedly led to an underestimation of the anxiety-inducing effects of pentagastrin and led to limitations in the conclusions that can be drawn regarding the anxiogenic effects of pentagastrin in social phobics per se. Nevertheless, pentagastrin led to significant increases in anxiety when compared to placebo, and only the combination of pentagastrin and social interaction led to panic attacks.

It is of interest to note that the social interaction task was equally anxiogenic in patients with social phobia and patients with panic disorder. Because the presence of comorbid social phobia in three of the patients with panic disorder could potentially have led to this observation, data was reanalyzed omitting these three patients. Results from this reanalysis were not significantly different from the original analysis (although standard errors were slightly increased in the panic disorder group, secondary to the smaller group size).
PLACEBO
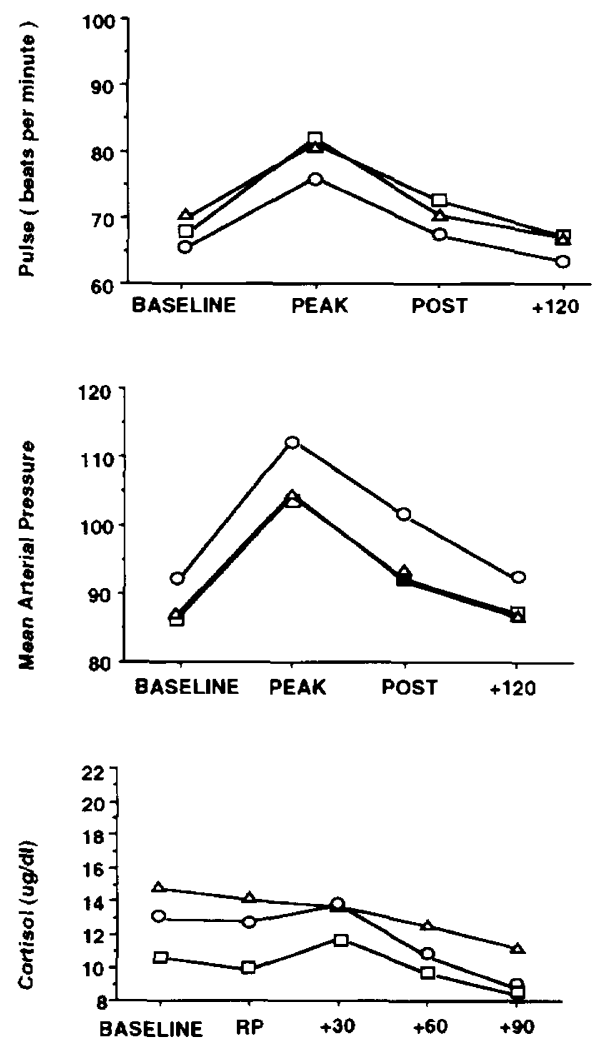

TIME
PENTAGASTRIN
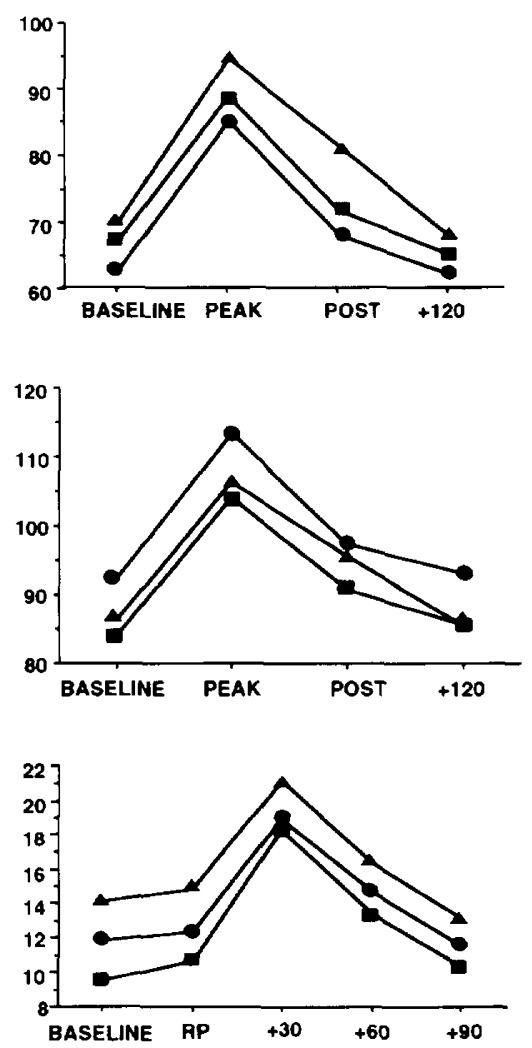

TIME
Figure 2. Cardiovascular and cortisol measures in patients with social phobia, patients with panic disorder, and healthy volunteers following infusions of placebo and pentagastrin. Peak, group mean maximum values during the 5-minute social interaction task/infusion procedure. Post, group mean values immediately following the social interaction task. $R P$, cortisol values from blood samples collected immediately following pentagastrin infusion during the role-play (averaged for each group). Triangles, panic disorder $(n=11)$; circles, social phobia $(n=19)$; squares, controls $(n=19)$. 
It could be argued that the use of the social interaction task, by increasing anxiety in patients with social phobia, created a situation in which only a small (and possibly nonspecific) increase in anxiety could lead to panic (i.e., by increasing "baseline" anxiety, the percentage of patients who reported panic attacks increased). However, using that logic, one would then expect the social interaction task to increase the likelihood of panic attacks in patients with panic disorder, as patients with panic disorder were equally anxious as patients with social phobia following the social interaction task. Instead, rates of panic attacks in patients with panic disorder in this study are almost identical (or slightly lower) than those found in previous studies, suggesting that elevations in anxiety produced by the social interaction task did not increase the likelihood for panic in that group and that mere elevations in baseline anxiety do not suffice in facilitating pentagastrin-induced panic attacks. Furthermore, preliminary results from an ongoing study in patients with social phobia and panic indicate that pentagastrin, when administered during late stage 2 sleep can lead to panic attacks ( $2 / 2$ patients with social phobia and $3 / 4$ patients with panic disorder have panicked to date), again suggesting a vulnerability to pentagastrin-induced panic in patients with social phobia.

Although the present findings are largely consistent with those previously found using CCK-4 and pentagastrin in panic disorder, there also are some inconsistencies. For example, patients generally found that pentagastrin-induced symptoms were not similar to their naturally occurring symptoms. More commonly, patients were able to identify some symptoms (e.g., sweating, stomach discomfort) that were characteristic of their typical experience, but also symptoms that were not typical features of their naturally occurring anxiety. In addition, patients with social phobia generally reported that pentagastrin-induced symptoms were more intense than those usually experienced. Both patient groups reported that pentagastrin-induced anxiety was more abrupt and of shorter duration than their naturally occurring anxiety, as has been previously reported in panic disorder (Bradwejn et al. 1990; Abelson and Nesse 1994; van Megen et al. 1994), with symptoms reaching a peak and resolving within the 31 /2-minute period during and following drug infusion. Pentagastrin infusion did not generally lead to pronounced respiratory symptoms, and for patients with panic disorder in whom shortness of breath is a prominent panic attack feature, this component was not typically reproduced. Differences in the present study, compared to previous studies using pentagastrin, might be due to the fact that pentagastrin (and placebo) was administered over a 60-s period, whereas in previous studies, drug was given over less than $60 \mathrm{~s}$ (Abelson and Nesse 1994a; Abelson et al. 1994) or as a bolus (van Megen et al. 1994).
Cortisol increases following pentagastrin were similar to those that have been previously reported using pentagastrin in patients with panic disorder and healthy volunteers (Abelson and Nesse 1994; McCann et al. 1995). Although these increases were significant, they did not differentiate patients with anxiety disorders and healthy controls and could either be due to the pharmacological effects of pentagastrin or to the experience of unpleasant physical and emotional symptoms associated with pentagastrin infusion.

Although the present findings support the view that the anxiogenic effects of CCK-4 and pentagastrin are mediated through actions at CCK receptors, the location of these receptors is not clear. It is unlikely that significant concentrations of pentagastrin enter the brain to act at CCK receptors in the limbic system, cerebral cortex, or basal ganglia, although small amounts may gain access to the brain via the area postrema or nucleus tractus solitarius. It is more likely that CCK receptors located on the vagus nerve or in the gastrointestinal tract are stimulated, and that these peripheral CCK receptors relay signals to brain regions implicated in anxiety. Furthermore, activation of peripheral CCK receptors themselves is probably important for some of the panic attack symptoms produced by CCK and pentagastrin (e.g., stomach discomfort).

Although the present results suggest that there may be common neurobiological substrates involved in the pathophysiology of social phobia and panic disorder, there also are obvious differences between the two disorders. For example, by definition, the phenomenology of panic disorder and social phobia is distinct, and, as mentioned, three previous comparisons of the two disorders found differences between the two patient populations. What the present findings do suggest, however, is that CCK agonists may lead to activation of a common anxiogenic substrate shared in social phobia and panic disorder. For example, it is possible that pentagastrin acts at CCK receptors located on the vagus nerve, with subsequent activation of brain centers responsible for panic (e.g., locus ceruleus, amygdala, cerebral cortex). Thus, although pentagastrin may lead to the activation of a final common pathway shared by the two disorders, the naturally occurring mechanisms for anxiety in the two populations could be quite distinct.

In conclusion, findings from the present study indicate that patients with generalized social phobia, like those with panic disorder, have similar rates of pentagastrin-induced panic attacks when pentagastrin is administered in the context of a social interaction task. Furthermore, when administered using this paradigm, patients with panic disorder and social phobia cannot be distinguished based on measures of anxiety. The striking similarities between patients with panic disorder and those with generalized social phobia found in the present study underscore the importance of contex- 
tual variables in pharmacological challenges and suggest an overlap in the neurobiology of panic disorder and social phobia.

\section{ACKNOWLEDGMENTS}

We would like to thank the following individuals who served as confederates in the structured role-play and without whom this research would not have been completed: Lisa Malloy, Cecilia Mermel, Mark Jones, Omar Ali, Elizabeth Disney, Melissa Spearing, Melissa Mertl, Mark Willis, Melanie Dubin, and Rachel Hayden. In addition, we would like to thank Dr. Robert M. Post for his support of this research, Dr. John Bartko for statistical consultation, and Dr. Jacqueline Crawley and Dennis Murphy for their thoughtful reviews of this manuscript.

\section{REFERENCES}

Abelson JL, Nesse RM (1994): Pentagastrin infusions in patients with panic disorder I. Symptoms and Cardiovascular Responses. Biol Psychiatry 36:73-83

Abelson JL, Nesse RM, Vinik AI (1994): Pentagastrin infusions in patients with panic disorder II. Neuroendocrinology. Biol Psychiatry 36:84-96

Bradwejn J, Koszycki D, Meterissian (1990): Cholecystokinin-tetrapeptide induced panic attacks in panic disorder. Can J Psychiatry 35:83-85

Bradwejn J, Koszycki D, Bourin M (1991a): Dose ranging study of the effect of CCK-4 in healthy volunteers. J Psychiatr Neurosci 16(2):91-95

Bradwejn J, Koszycki D, Payeur R (1991b): Enhanced sensitivity to cholecystokinin-tetrapeptide in panic disorder: Clinical and behavioral findings. Arch Gen Psychiatry 48:603-610.

Crawley JN (1985): Comparative distribution of cholecystokinin and other neuropeptides. Ann NY Acad Sci 448:1-8

Ervin FR, Palmour RM, Bradwejn J (1991): A new primate model for panic disorder. Biol Psychiatry 29:430S.

Fekete M, Lengyel A, Hegedus B, Penke B, Zarandy M, Toth GK, Telegdy G (1984): Further analysis of the effects of cholecystokinin octapeptide on avoidance in rats. Eur J Pharmacol 98:79-91

Fyer AJ, Mannuzza S, Gallops MS, Martin LY (1990): Familial transmission of simple phobias and fears: A preliminary report. Arch Psychiatry 47:252-256

Fyer AJ, Mannuzza S, Chapman TF, Liebowitz MR, Klein DF (1993): A direct interview family study of social phobia. Arch Gen Psychiatry 50:286-293

Harro J, Vasar E (1991): Evidence that CCK-B receptors mediate the regulation of exploratory behavior in the rat. Eur J Pharmacol 193:379-381

Harro J, Vasar E, Bradwejn J (1993): Cholecystokinin in animal and human research on anxiety. Trends Pharmacol Sci 14:244-249
Kendler K, Neale M, Kessler R, Heath AC, Eaves LJ (1992): The genetic epidemiology of phobias in women: The interrelationship of agoraphobia, social phobia, situational phobia and simple phobia. Arch Gen Psychiatry 49:273-281

Kessler RC, McGonagle DK, Zhao S, Nelson CB, Hughes M, Eshleman S, Wittchen HU, Kendler KS (1994): Lifetime and 12-month prevalence of DSM-III-R psychiatric disorder in the United States: Results from the National Comorbidity Survey. Arch Gen Psychiatry 51:8-19

Kendler KS, Walters EE, Neale MC, Kessler RC, et al. (1995): The structure of the genetic and environmental risk factors for six major psychiatric disorders in women. Phobias, Generalized anxiety disorder, panic disorder, bulimia, major depression and alcoholism. Arch Gen Psychiatry 52(5):374-83

Liebowitz MR, Fyer AJ, Gorman JM, Dillon D, Davies S, Stein JM, Cohen BS, Klein DF (1985): Specificity of lactate infusions in social phobia versus panic disorders. Am J Psychiatry 142(8): 947-949

McCann UD, Slate SO, Geraci M, Uhde TW (1995): Peptides and anxiety: A dose response evaluation of pentagastrin in healthy volunteers. Anxiety 1:258-267

Regier DA, Boyd JH, Burke JD, Rae DS, Myers JK, Kramer M, Robins LN, George LK, Karno M, Locke BZ (1988): Onemonth prevalence of mental disorders in the United States: Based on five Epidemiologic Catchment Area sites. Arch Gen Psychiatry 45:977-986

Schleyer B, Aarons C, Mannuzza S, Martin L, Fyer AJ (1990): Schedule for Affective Disorders and Schizophrenia Lifetime Version Modified for the Study of Anxiety Disorders (Updated for DSM-III-R).

Speilberger CD, Gorsuch RW, Lushene RE (1970): State-Trait Anxiety Inventory. Palo Alto, CA, consulting Psychologists Press, Inc.

Tancer ME, Stein MB, Uhde TW (1991): Lactate response to caffeine in panic disorder: A replication using an "anxious" control group. Biol Psychiatry 29:57

Tancer ME, Stein MB, Uhde TW (1994): Growth hormone response to intravenous clonidine in social phobia: Comparison to patients with panic disorder and healthy volunteers. Biol Psychiatry 34:252-256

Uhde TW (1990): Caffeine provocation of panic: A focus on biological mechanisms. In Ballenger JC (ed), Neurobiology of Panic Disorder, Frontiers in Clinical Neuroscience, vol. 8. New York, Alan R Liss

Uhde TW, Tancer ME, Black B, Brown TM (1991): Phenomenology and neurobiology of social phobia: Comparison with panic disorder. J Clin Psychiatry 52(11, suppl):31-40

van Megen HJGM, Westenberg HGM, den Boer JA, Haigh JRM, Traub M (1994): Pentagastrin induced panic attacks: Enhanced sensitivity in panic disorder patients. Psychopharmacology 114:449-455

Zung WWK (1971): A rating instrument for Anxiety Disorders. Psychosomatics 12:371-379 